\title{
APPLICATION OF A PREDICTION METHODOLOGY FOR MEMS RELIABILITY IN SHOCK ENVIRONMENTS
}

\author{
M. Naumann ${ }^{1 *}$,D. Lin ${ }^{2}, A . M c N e i l^{2}, G$. Li $^{2}$ and J. Mehner ${ }^{l}$ \\ ${ }^{1}$ Chemnitz University of Technology, Germany \\ ${ }^{2}$ Freescale Semiconductor, Inc., USA
}

\begin{abstract}
The paper investigates an approach to predict shock induced failure mechanisms of breakage and stiction at MEMS devices. The approach is applied at two surface micro-machined test devices which are exposed to shock accelerations up to $56,000 \mathrm{~g}$ $\left(1 \mathrm{~g}=9.81 \mathrm{~m} / \mathrm{s}^{2}\right)$. Observed failures are correlated to predicted failures in order to validate the proposed methodology. Predictions are based on a comparison between calculated loads obtained from numerical models and permitted loads of utilized device components. Therefore, a detailed characterization of the applied fabrication technology regarding both failure mechanisms is required. The paper presents a selection of data required to predict failures for both test devices.
\end{abstract}

\section{INTRODUCTION}

The adoption of MEMS devices within various automotive, medical and consumer markets products is determined by economical, functional as well as reliability aspects. MEMS devices are required to fulfill their functionality even under harsh environmental conditions. Common sources of failure are mechanical shocks on devices consisting of movable structural components. Depending on the load characteristics, two major failure mechanisms of breakage and stiction can be triggered [1].

As a common practice, rigid travel stops are applied to avoid excessive deflections during overload situations. This often produces very high impact loads at the stops as movable structural MEMS components are stopped almost immediately when they hit a stop. Excessive impact loads can cause breakage of stops and structural components. Furthermore, the probability of stiction at travel stops is increased by higher impact forces as demonstrated in [2]. Latest investigations have indicated that an improvement of device reliability is possible by introducing flexible stops to reduce arising impact loads [3], [4].

A new approach to evaluate the reliability of MEMS devices in shock environments was first proposed in [2]. The approach is intended to assess arising failures at MEMS devices which are exposed to shock accelerations by calculation of their transient system response including arising load conditions. A conclusion on device reliability requires the knowledge of permissible loads which have to be obtained from a detailed characterization of the utilized fabrication technology. The results in terms of fracture strength and adhesion behavior are compared to calculated loads in order to evaluate and predict the device reliability. Once the fabrication technology is characterized, the introduced methodology can be applied to arbitrary designs. The reliability of new MEMS products can be assessed and their design optimized even before first prototypes are fabricated which can be used to experimentally validate device reliability. Furthermore, the methodology is able to improve experimental reliability approaches as device specific critical load situations can be identified and tested purposefully.

In this paper the proposed approach is investigated by surface micro-machined test devices which have been exposed to shock accelerations up to $56,000 \mathrm{~g}$. The observed mechanism of failure and the failure probability are compared to the predictions of the reliability approach at different shock levels.

\section{TEST DEVICES}

Two different test devices have been exposed to shock accelerations parallel to the substrate in order to investigate both failure mechanisms of breakage and stiction.

Figure 1a) shows the SEM image of the test device which was used to investigate the fracture behavior of travel stops. The device consists of a seismic mass suspended at meander shaped springs.

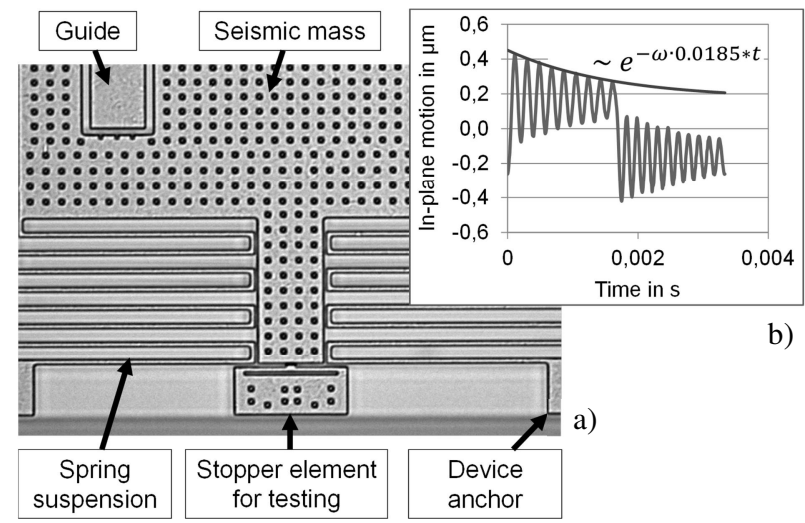

Figure 1: a) Surface micro-machined test device for breakage investigations; $b$ ) Determination of the damping ratio.

The overall dimension of the test device is $320 \mu \mathrm{m} \times 370 \mu \mathrm{m}$ which is small in order to maximize the number of devices on a single chip and therefore to increase the level of confidence of the obtained results. Furthermore, damping has been minimized in order to investigate dynamic impacts when critical loads arise primarily due to the deceleration of the proof mass [4]. The damping ratio of $\mathrm{dr}=0.018$ has been determined by measuring the in-plane decay process (Fig. 1b). Nonlinearities due to the squeeze effect can be neglected for this kind of test devices. Figure 2 shows a small selection of investigated stopper elements.

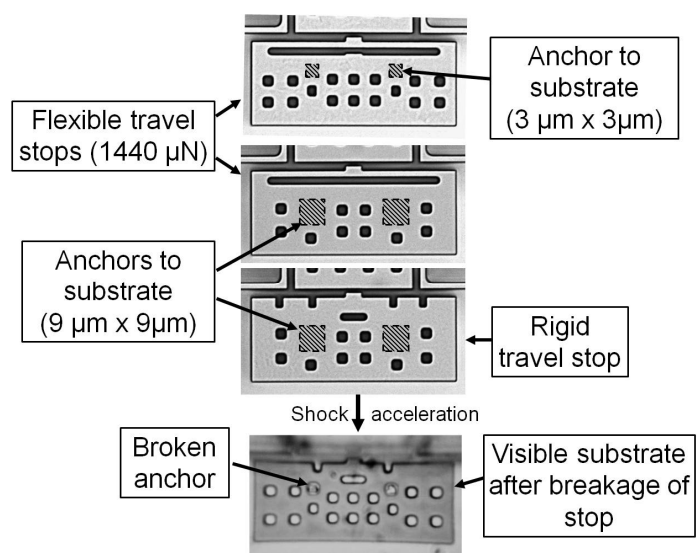

Figure 2: Stopper elements of different stiffness and anchor areas to substrate before and after breakage.

Solid-State Sensors, Actuators, and Microsystems Workshop Hilton Head Island, South Carolina, June 3-7, 2012 
Flexible travel stops of type 720, 1440 and 4350 have been compared to rigid travel stops without any additional flexibility. The type reference of flexible stops corresponds to their stiffness with the unit in $\mathrm{N} / \mathrm{m}$. Each kind of stop has been combined with three different types of anchors to substrate $3 \times 3,5 \times 5$ and $9 \times 9$ corresponding to total anchor areas per stop of $18 \mu \mathrm{m}^{2}, 50 \mu \mathrm{m}^{2}$ and $162 \mu \mathrm{m}^{2}$, respectively.

\section{PROCESS CHARACTERIZATION}

\section{Breakage}

During the applied shock accelerations, stopper elements of the test devices are exposed to forces parallel to the substrate causing a combination of shearing and bending loads. Breakage will occur at the anchors if the load exceeds their fracture strength. In order predict failures for this kind of load condition, characterization results of a special MEMS device have been utilized. The device was used to determine the fracture strength of stopper elements exposed to the described load condition for different anchor areas by static measurements. Figure 3 illustrates the results. Statements on the reliability of a specific stopper element can directly be achieved by comparing the output of the impact models in terms of calculated impact forces with the corresponding measured fracture forces.

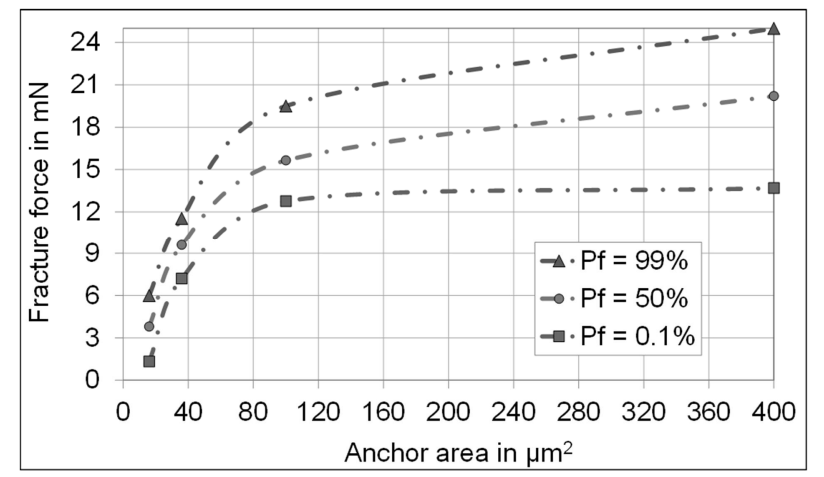

Figure 3: Fracture strength depending on the anchor area for different probabilities of failure.

A closer look on the measurement data revealed the brittle fracture behavior of anchors. Hence, the Weibull distribution was applied to mathematically describe the probability of failure depending on the load. Figure 4 shows the fracture data and the fitted three-parameter Weibull distribution for two anchor sizes of $36 \mu \mathrm{m}^{2}$ (type 6x6) and $100 \mu \mathrm{m}^{2}$ (type 10x10).
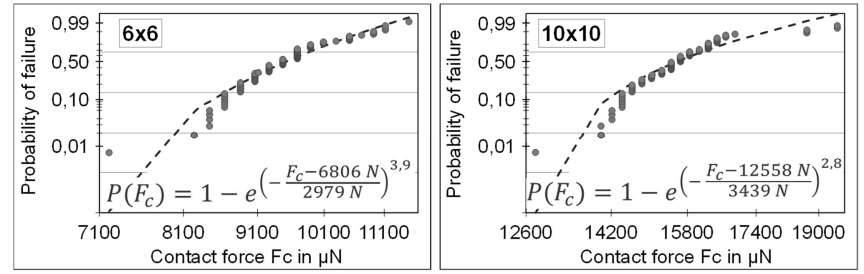

Figure 4: Description of the fracture behavior of anchors of type $6 \times 6$ and $10 \times 10$ by the tree-parameter Weibull distribution.

The diagrams verify the applicability of the Weibull distribution to describe the real fracture behavior of anchors with adequate accuracy. However, the validity of the Weibull theory still needs to be proven as the current evaluation of data links the event of fracture to the acting stopper force without any consideration on the load distribution along the anchor required to determine Weibull parameters like the characteristic stress.

\section{Stiction}

Contacts to travel stops at the test devices occur at side walls of the functional layer as shock accelerations are applied parallel to substrate. The required characterization of adhesion forces to side walls has been performed by a special MEMS device which applied compressive contact forces to stopper elements by electrostatic actuation. After each force step the quantity of sticking devices as well as arising adhesion forces have been determined. The results are illustrated in Figure 5.
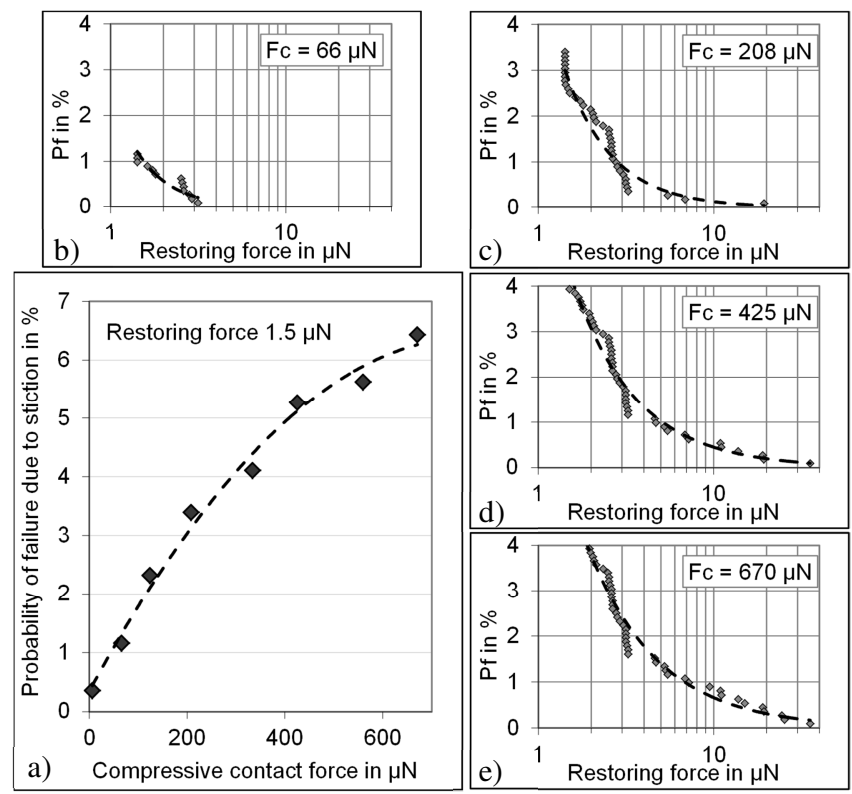

Figure 5: a) Failure rate due to stiction at a stopper element depending on the applied compressive contact force. b-e) Probability of failure Pf due to stiction depending on the restoring force of a device for applied compressive contact forces $F c$ of $66 \mu N(b), 208 \mu N(c), 425 \mu N(d), 670 \mu N(e)$.

In case of the investigated fabrication technology, increased contact forces lead to higher failure rates (Fig. 5a) and thus to larger adhesion forces (Fig. 5b-e). For example, the failure rate of a MEMS device with a restoring force of $5 \mu \mathrm{N}$ would be about $0.5 \%$ after contact to a travel stop with a compressive force Fc of about $208 \mu \mathrm{N}$. Below $\mathrm{Fc}=66 \mu \mathrm{N}$ most likely the device would not stick at all. At $\mathrm{Fc}=425 \mu \mathrm{N}$ the failure rate increases up to about $1 \%$. For contacts with a compressive force of $670 \mu \mathrm{N}$ the failure rate is about $1.5 \%$.

A further investigation on the dependency of adhesion forces on the number of performed contact cycles revealed an increase of adhesion forces by about $20 \%$ after the first cycle. $32 \%$ of the detected sticking incidences are affected by compressive contact forces above $124 \mu \mathrm{N}$.

\section{IMPACT MODEL}

The transient system responses of the test device exposed to shock accelerations are calculated by a lumped element impact model solving the differential equation of motion of the system. The model accounts for nonlinear interactions of the proof mass with travel stops including the resulting adhesion forces and the 
possible event of stiction failure. Figure 6 illustrates the approach. System parameters like device mass and damping as well as the measured shock accelerations are used as input parameters.
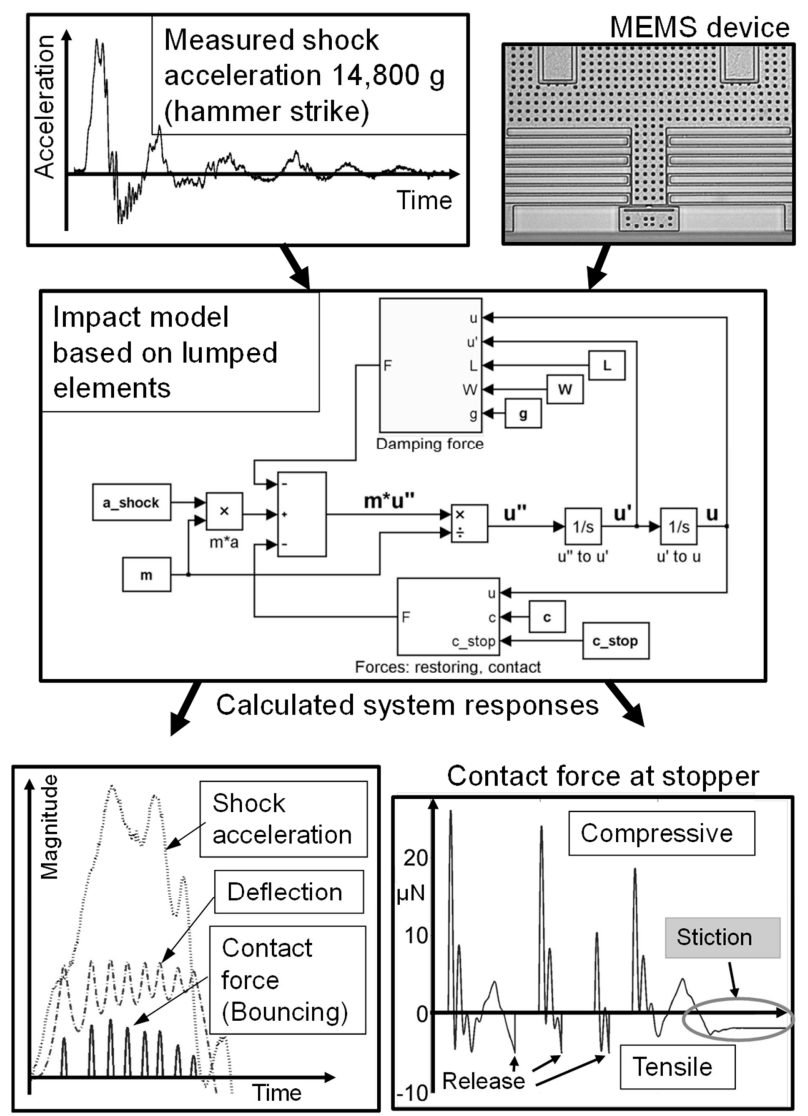

Contact force at stopper

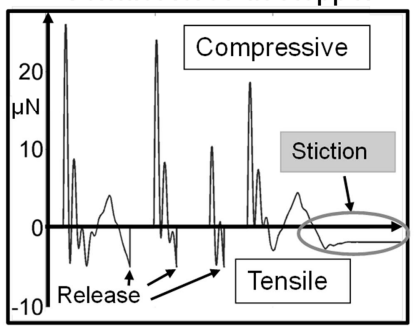

Figure 6: Calculation of the transient system response of the test device for breakage characterization exposed to a measured shock acceleration.

\section{SHOCK TESTS}

\section{Procedure}

Shock tests have been performed for diced silicion chips which have been mounted on a special chip carrier by conductive glue. The applied shock levels have been measured by a reference accelerometer (B\&K 8309) which has been mounted on the chip carrier. In total, three test runs have been performed whereas a hammer strike procedure (run 1 and 2) and a Hopkinson Bar have been utilized. At each test run a series of shocks have been applied to the chip carrier. The shock level has been increased stepwise form low g-level (about 6,000 g) to high g-level (up to 56,000 g). Arising failures have been recorded after each shock from 300 up to 1800 mounted devices.

\section{Breakage}

Figure 7 shows the obtained results in terms of failure rates depending on the applied shock level for different anchor types 3x3 (Fig. 7a), 5x5 (Fig. 7b, 7d) and 9x9 (Fig. 7c). Therefore, the efficiency of different flexible travel stops becomes obvious. For example, considering the results of anchors of type $3 \times 3$ at $14,900 \mathrm{~g}$ (Fig. 7a). The failure rate of rigid travel stops already reached $100 \%$ whereas the failure rate of the flexible stops 720 is only $6 \%$. Similar results can be observed at anchors of types $5 \times 5$ and $9 \times 9$.
The optimum stiffness of flexible stops depends on the utilized anchor area and has been detected to $720 \mathrm{~N} / \mathrm{m}$ of type $3 \times 3$, $1440 \mathrm{~N} / \mathrm{m}$ of type $5 \times 5$ and $4350 \mathrm{~N} / \mathrm{m}$ of type $9 \times 9$.
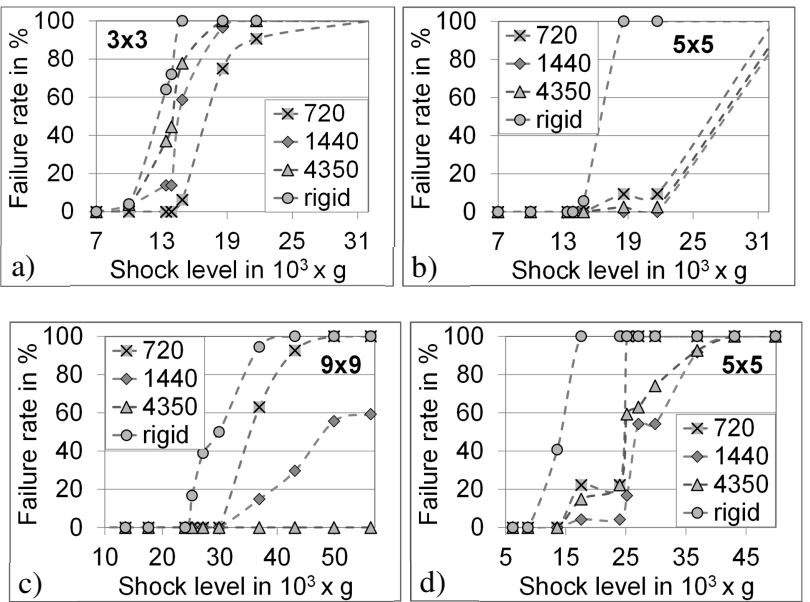

Figure 7: Failure rates of stopper elements of different stiffness and anchor type depending on the applied shock level; anchor types: a) $3 \times 3, b) 5 \times 5$, c) $9 \times 9$, d) $5 \times 5$

A comparison of results between the test runs reveals small differences which can be explained by different shock characteristics in terms of superimposed oscillations and the corresponding frequencies (resulting into deviant loads on the stops for comparable maximum shock levels). Figure $7 \mathrm{~b}$ and $7 \mathrm{~d}$ enable a comparison of results between test run $1(7 \mathrm{~b}$, hammer strike) and test run 3 (7d, Hopkinson Bar) for anchors of type $5 \times 5$. However, the efficiency of flexible travel stops has been verified by all test runs as general trends are comparable.

Shock levels which have been identified to cause damage at stopper elements of the test devices seem to be quite high ranging from $8,000 \mathrm{~g}$ up to $56,000 \mathrm{~g}$. For real MEMS products like gyroscopes or accelerometers, much lower shock accelerations will result into similar failure modes as masses are higher. Therefore, larger anchor size is needed to counter balance for larger mass.

\section{Stiction}

Figure 8 illustrates the observed failure rates due to stiction as a result of the applied shock acceleration for test run 3. For loads up to $30,000 \mathrm{~g}$, the number of sticking devices increases with shock acceleration. For loads greater than $30,000 \mathrm{~g}$, the failure rate remains at an almost constant high level of about $4.6 \%$.

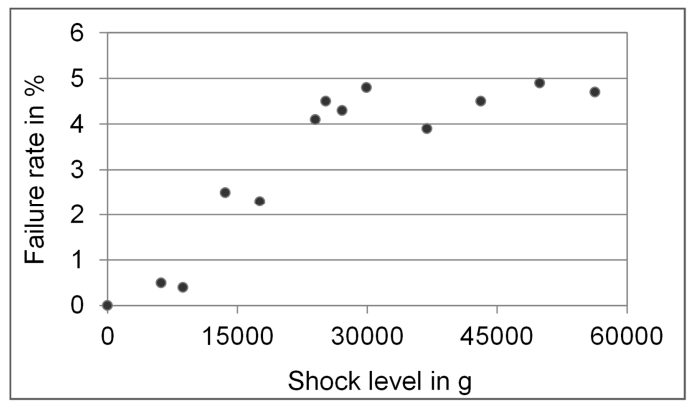

Figure 8: Failure rate due to stiction depending on the applied shock acceleration. 


\section{APPLICATION OF THE IMPACT MODEL Breakage}

The introduced impact model has been used to calculate the transient system responses of the test devices depending on the measured shock accelerations. From the solutions the maximum impact forces on the test stopper elements have been extracted. Figure 9a shows the calculated impact forces for test run 3 at the 9x9 stopper version. Based on the knowledge of the fracture behavior of the utilized anchors the probability of failure has been calculated and compared to the measurement results (Fig. 9b). The results of the impact model represent the general failure trend. However, they do not match the measured behavior exactly which has been observed at all test runs.
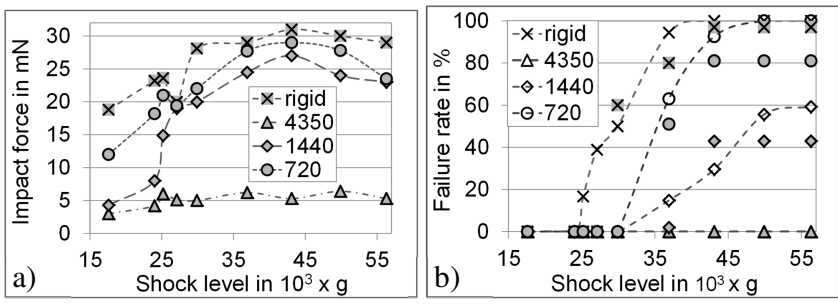

Figure 9: a) Calculated impact forces at stopper type 9x9 for test run 3; b) Comparison between observed and calculated failure rates (filled markers for calculated rates).

The reason for the deviations can be explained by a closer look at the system behavior. Figure 10a illustrates the measured shock acceleration of $43,000 \mathrm{~g}$ at the front side of the Hopkinson Bar (test run 3). Each time the material wave reaches the front side of the bar it is reflected creating an acceleration pulse and superimposed oscillations form the test setup. The calculated impact forces at test stops are illustrated in Figure 10b comparing the flexible stop 4350 with a rigid one. The resulting impact forces are reduced by the flexible stop from up to $31 \mathrm{mN}$ to about $5 \mathrm{mN}$ which correlates to the findings of the shock tests. At $43,000 \mathrm{~g}$ $100 \%$ of the rigid travel stops have been damaged compared to $0 \%$ for type 4350 (compare Fig. 9b).

Due to the low damping and the oscillations of the acceleration load, excessive bouncing occurs between the travel stops of the test devices. For both test setups of hammer strike and Hopkinson Bar, it has been found that maximum impact forces occur during the bouncing process in case when the bouncing frequency matches one of the frequencies contained in the acceleration signal.

A detailed analysis of the bouncing history has shown convergence for a sufficient small simulation time step. However, the consideration of process variations which affect device parameters like mass and stiffness of stopper elements has a significant effect on the time response demonstrated in Figure 10c. The diagram compares calculated impact forces for two versions of rigid travel stops at a shock acceleration of $27,000 \mathrm{~g}$. A maximum impact force of $23.6 \mathrm{mN}$ has been determined for the original stop leading to a predicted failure rate of $0 \%$ compared to $39 \%$ of observed failure rate (Fig. 9b). The deviation can be explained by possible process variations. A reduction of the stopper stiffness by $10 \%$ would increase the impact force to $33 \mathrm{mN}$ which corresponds to a failure rate of $100 \%$. Hence, the strong dependency of the bouncing response on the device parameters can explain the difference between predicted and detected failure rates.

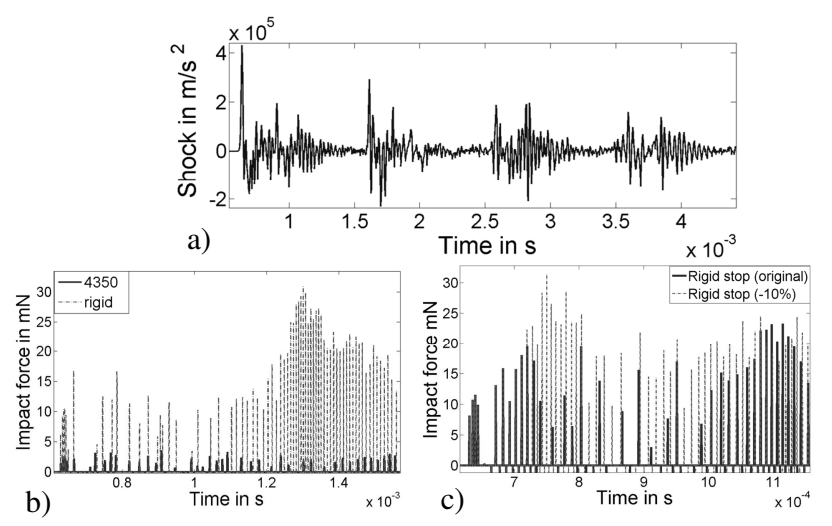

Figure 10: a) Measured shock acceleration (43.000 g) b) Comparison of impact forces between a flexible stop (4350) and a rigid one. c) Comparison of impact forces at rigid stops with $10 \%$ deviation.

\section{Stiction}

Device failure due to stiction occurs when adhesion forces at the travel stops exceed the tensile forces from the spring suspension trying to restore the initial device position. Additional tensile force at the travel stops can arise from the external shock acceleration as well as from the bouncing process. The analysis of the calculated system responses of the utilized test device at different g-levels has shown that stiction is very unlikely as long as the external shock acceleration lasts as tensile forces exceed minimum $100 \mu \mathrm{N}$. First sticking incidences occur in case when the external shock acceleration and bouncing almost decayed. Predicted failure rates range from $1 \%$ up to $3.1 \%$.

\section{SUMMARY}

The paper has demonstrated the ability to describe the observed failures for the test devices by application of the introduced approach for MEMS reliability with adequate accuracy. Limitations result from the unknown distributions of device parameters due to process variations such as mass and stiffness variations which have a significant effect on calculated system responses.

\section{REFERENCES}

[1] V. T. Srikar, S. D. Senturia, "The Reliability of Microelectromechanical Systems (MEMS) in Shock Environments", Journal of Microelectromechanical Systems, 11, (2002), pp. 206 - 214.

[2] M. Naumann, D. Lin, J. Mehner, A. McNeil, T. F. Miller, "Design evaluation of shock induced failure mechanisms of MEMS by correlation of numerical and experimental results", Proc. Transducers, 16, (2011), pp. 2891 - 2894.

[3] S. W. Yoon, "Vibration isolation and shock protection for MEMS", Dissertation, Michigan, 2009.

[4] M. Naumann, D. Lin, J. Mehner, T. Miller, "Design and Application of Flexible Stops for MEMS Devices", Proc. Sensors, 9, (2010), pp. 168 - 173.

\section{CONTACT}

*M. Naumann; michael.naumann@etit.tu-chemnitz.de 